\title{
Multimodality imaging for cardiac lymphoma
}

\author{
Joseph C. Lee ${ }^{1,2}(1) \cdot$ Yi-Tung Tom Huang ${ }^{3,4}$
}

Received: 6 August 2019 / Accepted: 16 October 2019 / Published online: 30 October 2019

(c) Japanese Society of Echocardiography 2019

We were very impressed with the work of Shikuma et al. [1] in their approach to this tumor, with unusual appearance, later proved to be cardiac lymphoma comprising large B cells. We also advocate for utilising multiple imaging modalities in such an instance. A single imaging modality is often simply not able to provide a comprehensive assessment. The different modalities provide information critical to the diagnosis and management of these uncommon tumours - which have a poor prognosis. This concurs with the tenets advocated by Mato et al. [2].

As mentioned, transthoracic echocardiography (TTE) demonstrated multiple nodules in the.thickened left ventricular wall with diffuse mild hypokinesis and moderate amount of pericardial effusion [1]. TTE was also helpful in this situation to evaluate the cardiac dysfunction it can additionally provide information regarding quantitative indices of the left and right ventricular function and valvular dynamics (if compromised) [3].

Positron emission tomography combined with computed tomography (PET-CT) was not utilised in this case but certainly could be of additional value in such a scenario. While only capable of lower resolution, it can also emphasize disease sites not clearly defined with (CT and MRI) tomographic imaging such as in assessment of lymph nodes borderline for significance on size criteria alone.

Determining the ideal biopsy site (and avoiding futile procedures) is another possible additional role of PET-CT [3]. In this instance, the biopsy from the inguinal lymph node was fruitful, fortunately. PET-CT would be helpful to determine which of the remainder would be most viable if the inguinal lymph node biopsy was non-diagnostic.

Joseph C. Lee

Joseph.Lee@health.qld.gov.au

1 Department of Medical Imaging, The Prince Charles Hospital, Chermside, QLD 4032, Australia

2 Faculty of Medicine, University of Queensland, Herston, QLD 4006, Australia

3 Department of Medical Imaging, Gold Coast University Hospital, Southport, QLD 4215, Australia

4 School of Medicine, Griffith University, Southport, QLD 4222, Australia
PET-CT is not universally available, however. It is much more accessible than, say, a decade earlier, but it is still mostly confined to tertiary referral centres and private imaging facilities in metropolitan areas. Many people are referred from regional and rural locations to make use of this technology.

MRI, in restaging of lymphoma, may continue to demonstrate contrast enhancement (possibly related to residual inflammation or scar) even though the tumor is no longer metabolically active [3]. Likewise, TTE may show the tumour is smaller but not completely resolved. Although TTE has an advantage over CT (as well as MRI and PETCT) in being less logistically difficult [4], PET-CT can give a better indication of the metabolic activity (rather than anatomical definition) and thus, determine whether the tumour is active and if it requires further treatment.

Thus, the case presented with an unusual manifestation as headlined in the title-reminds us of combining anatomical with functional assessment. PET-CT is often particularly helpful in our experience especially in determining the site of biopsy for diagnostic purposes and for post-treatment follow-up.

\section{References}

1. Shikuma A, Sawada T, Uchiyama H, et al. Cardiac involvement of malignant lymphoma presenting intra-ventricular-wall nodules. J Echocardiogr. 2018;. https://doi.org/10.1007/s12574-018-0411-7.

2. Mato AR, Morgans AK, Roullet MR, et al. Primary cardiac lymphoma: utility of multimodality imaging in diagnosis and management. Cancer Biol Ther. 2007;6:1867-70.

3. Lee JC, Platts DG, Huang YT, et al. Positron emission tomography combined with computed tomography as an integral component in evaluation of primary cardiac lymphoma. Clin Cardiol. 2010;33:E106-E108108.

4. Ochi H, Hozumi T, Yamamoto M, et al. Cardiac involvement of malignant lymphoma and its improvement after chemotherapy. $\mathbf{J}$ Echocardiogr. 2013;11:38-9.

Publisher's Note Springer Nature remains neutral with regard to jurisdictional claims in published maps and institutional affiliations. 